\title{
Manipulating complex network structures in virtual reality and 3D printing of the results
}

\author{
Alberto Betella \\ SPECS, NRAS \\ Universitat Pompeu Fabra \\ Barcelona, Spain
}

\author{
Alex Escuredo \\ SPECS, NRAS \\ Universitat Pompeu Fabra \\ Barcelona, Spain
}

\author{
Enrique Martínez \\ SPECS, NRAS \\ Universitat Pompeu Fabra \\ Barcelona, Spain
}

\author{
Pedro Omedas \\ SPECS, NRAS \\ Universitat Pompeu Fabra \\ Barcelona, Spain
}

\author{
Paul F.M.J. Verschure \\ SPECS, NRAS \\ Universitat Pompeu Fabra \\ and ICREA \\ Barcelona, Spain
}

\begin{abstract}
We present an immersive VR system that allows to manipulate network data structures by creating, removing or reconfiguring their elements and export the results at any time for direct 3D printing.
\end{abstract}

\section{INTRODUCTION}

Although its first appearance on the market can be tracked up to three decades ago, stereolithography (also known as $3 \mathrm{D}$ printing) has become available to the general public just in recent years. The more affordable prices, along with the free and open-source licenses under which most of the hardware and software specifications for 3D printing are released, led to an unprecedented growth and diffusion of these techniques.

One of the main advantages of 3D printing is the possibility to create rapid prototypes. Rapid prototyping had been previously employed mainly in the industry sector, however it has recently extended to a wide range of scientific fields, including (but not limited to) robotics, medicine and neuroscience, giving rise to new exciting opportunities.

$3 \mathrm{D}$ printing can be coupled to virtual reality (VR) environments to provide new tools for the exploration and manipulation of complex data. Immersive VR techniques can enhance the understanding of complex multi-dimensional information. By adding a physical outcome to these VR systems, more comprehensive data visualization and exploration tools can be obtained. As a matter of fact, physical objects are immediate to manipulate and can offer a deeper

\footnotetext{
*Corresponding e-mail: paul.verschure@upf.edu
}

Pre-print version. The final version of this manuscript is available at http://doi.acm.org/10.1145/2617841.2620718

Laval Virtual VRIC'14, April 9-11, 2014 Laval, France

This is a pre-print version of the manuscript. Final version published by ACM. DOI: 10.1145/2617841.2620718. insight of the results [4], thus providing a further layer of understanding of the data.

We previously built an immersive VR application for the study of artificially generated neuronal networks [2]. Subsequently, we developed a new generalized version of the system to represent any data structure stored in form of graph. As a test case, we used the human connectome, the network of nodes and connections that defines the information flow in the brain. Our application allows to literally navigate through the data space and change its organization using a natural interaction paradigm [3].

Here we present a new feature of this system that allows to manipulate a subset of a complex connectome network by creating, removing or reconfiguring its elements and export the results of this process at any time for direct $3 \mathrm{D}$ printing.

\section{MATERIAL \& METHODS}

Our VR application is implemented in the eXperience Induction Machine (XIM), an immersive room equipped with a number of sensors and effectors including 4 projection screens, a luminous interactive floor, a sound system, a tracking system, as well as wearable and unobtrusive sensors [1].

The user's movements and gestures in the space are tracked using the Microsoft Kinect. In addition, fingers movement and forearm position are tracked using the sensing glove, which was custom-tailored for the XIM space [5]. We use 180 degrees immersive projections to visualize the dataset in $3 \mathrm{D}$. The nodes of the network are rendered as spheres connected through one or more edges, which are represented as tubes.

Our system is based on Unity3D $(\mathrm{C \# )}$ and can handle large networks with high performance. A human connectome dataset composed $1 \mathrm{k}$ nodes and $30 \mathrm{k}$ connections is rendered in real-time with a frame rate between 70 and 170fps (depending on the quality settings).

Here we present a novel feature of our application that allows users to select a data subset within the network, explore or reconfigure it by adding, removing or reorganizing its elements through natural gestures (Figure 1) and print the results in $3 \mathrm{D}$ at any time. To do so, the user can trigger a contextual graphical user interface by holding the left 


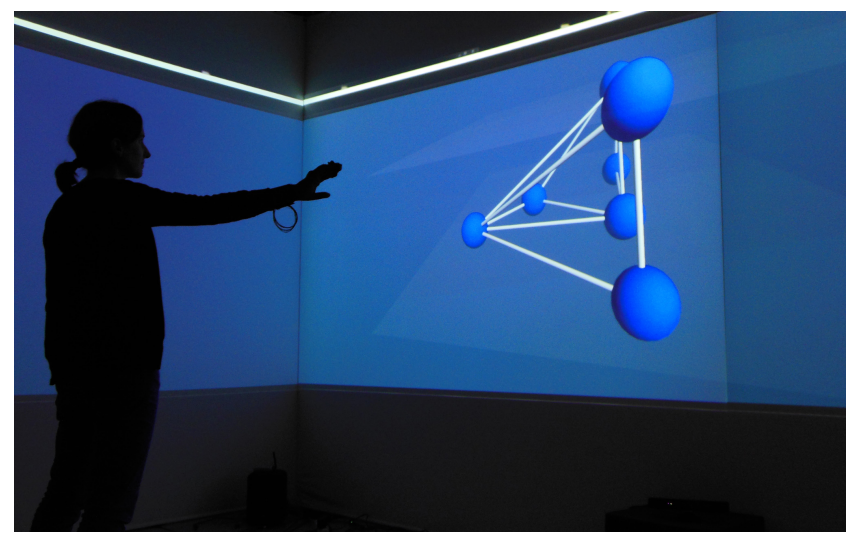

Figure 1: A user while manipulating in real time a subset of a complex network in the eXperience Induction Machine (XIM). The interaction is implemented using the Microsoft Kinect and the sensing glove. Through natural hand gestures, the user can create, remove and reconfigure nodes or edges and send the results to the $3 \mathrm{D}$ printer at any time.

hand towards the upper-left area of the virtual scenario and by selecting the "3D print" icon. This action produces in real-time a ready-to-print STL file in ASCII format containing the tessellated representation of the $3 \mathrm{D}$ scene. The generated file is subsequently sent through the SSH protocol to the local machine connected to a 3D printer, where a running daemon detects new files, runs the slicer through a batch process and prints the results in $3 \mathrm{D}$.

For the creation of the physical object (Figure 2) we use a Makerbot Replicator 2 3D Printer (MakerBot Industries, USA) that allows to obtain models with layer resolution up to 100 -micron and 410 cubic inches of volume.

\section{DISCUSSION}

We enhanced a previously built immersive VR system that allows to explore complex networks with the possibility of selecting subsets of data and exporting them in real time for $3 \mathrm{D}$ printing. This way, the user can create a set of physical objects that serve as "bookmarks" to relevant locations in the data space. This addition provides a further layer of exploration that can offer new insights on the data space.

Although the complexity of the network exported in STL format using our system in XIM is theoretically unlimited (i.e. limited only by computational power), hardware limitations in 3D printing technologies currently allow to produce physical networks composed of a small number of elements, mainly due to their thin edges (using our printer, we are able to successfully print up to 10 nodes and 20 connections in a single $3 \mathrm{D}$ piece).

Our next step will consist in the implementation of an algorithm to distribute the exported STL files into a series of clusters that can be printed separately as a workaround to the complexity issue. Nonetheless, we expect future hardware and software solutions for 3D printing to overcome the aforementioned limitations. Future work will consist in an empirical evaluation of the benefits added by physical 3D models, when compared to the immersive interaction alone.

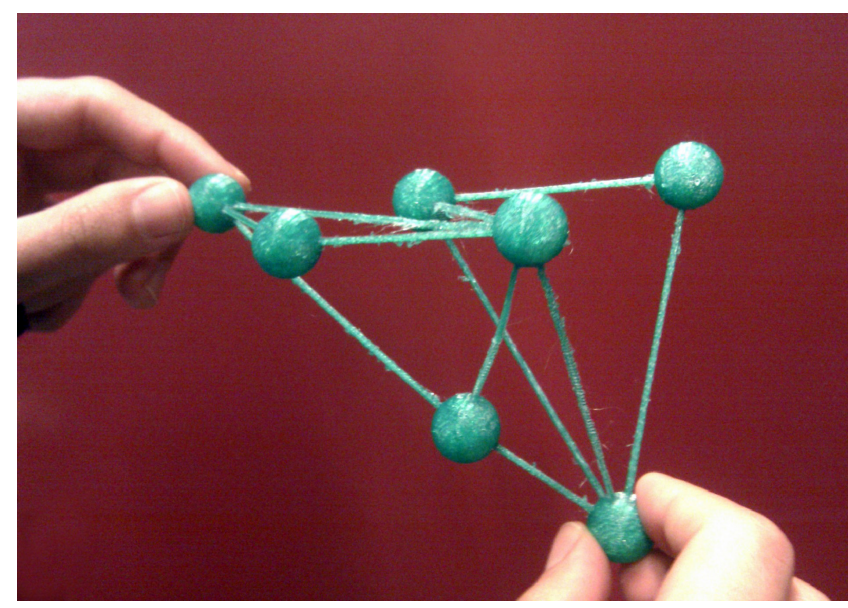

Figure 2: Example of a network subset generated in real-time in XIM (Figure 1) and printed in 3D.

\section{ACKNOWLEDGEMENT}

EU FP7-ICT-2009-5 grant agreement n. 258749 [CEEDS]. The Generalitat de Catalunya (CUR, DIUE) and the European Social Fund are supporting this research.

\section{REFERENCES}

[1] U. Bernardet, S. B. i. Badia, A. Duff, M. Inderbitzin, S. L. Groux, J. Manzolli, Z. Mathews, A. Mura, A. Väljamäe, and P. F. Verschure. The eXperience Induction Machine: A New Paradigm for Mixed-Reality Interaction Design and Psychological Experimentation. In E. Dubois, P. Gray, and L. Nigay, editors, The Engineering of Mixed Reality Systems,

Human-Computer Interaction Series, pages 357-379. Springer London, London, 2010.

[2] A. Betella, R. Carvalho, J. Sanchez-palencia, U. Bernardet, and P. F. M. J. Verschure. Embodied Interaction with Complex Neuronal Data in Mixed-Reality. In Virtual Reality International Conference (VRIC 2012), 2012.

[3] A. Betella, E. Martínez, R. Zucca, X. D. Arsiwalla, P. Omedas, S. Wierenga, A. Mura, J. Wagner, F. Lingenfelser, E. André, D. Mazzei, A. Tognetti, A. Lanatà, D. De Rossi, and P. F. M. J. Verschure. Advanced interfaces to stem the data deluge in mixed reality: placing human (un)consciousness in the loop. In ACM SIGGRAPH 2013 Posters, SIGGRAPH '13, pages 68:1-68:1, New York, NY, USA, 2013. ACM.

[4] C. H. Séquin. Rapid Prototyping: A 3D Visualization Tool Takes on Sculpture and Mathematical Forms. Commun. ACM, 48(6):66-73, June 2005.

[5] J. Wagner, F. Lingenfelser, E. André, D. Mazzei, A. Tognetti, A. Lanatà, D. D. Rossi, A. Betella, R. Zucca, P. Omedas, and P. F. M. J. Verschure. A sensing architecture for empathetic data systems. In Proceedings of the 4th Augmented Human International Conference, AH '13, pages 96-99, New York, NY, USA, 2013. ACM. 